\title{
Existence and asymptotic properties of positive solutions for a general quasilinear Schrödinger equation
}

Xiang Zhang ${ }^{1}$ and Yimin Zhang ${ }^{1,2^{*}}$

\section{"Correspondence:}

zhangym802@126.com

'Department of Mathematics,

Wuhan University of Technology,

Wuhan, P.R. China

${ }^{2}$ Center for Mathematical Sciences,

Wuhan University of Technology,

Wuhan, P.R. China

\begin{abstract}
By a change of variables with cut-off functions, we study the existence and the asymptotic behavior of positive solutions for a general quasilinear Schrödinger equation which arises from plasma physics. We extend the results of (Adv. Nonlinear Stud. 18(1):131-150, 2017) from $\alpha=1$ to $\alpha>\frac{1}{2}$. Especially, we can consider the exponent $p$ in $\left(2,2^{*}\right)$ for all $N \geq 3$.
\end{abstract}

Keywords: Quasilinear Schrödinger equation; Existence; Asymptotic properties

\section{Introduction}

In this paper, we study the existence and asymptotic behavior of positive solutions for the following general quasilinear elliptic equation:

$$
-\Delta u+V(x) u-\alpha \gamma\left(\Delta\left(|u|^{2 \alpha}\right)\right)|u|^{2 \alpha-2} u=|u|^{p-2} u, \quad x \in \mathbb{R}^{N},
$$

where $\alpha>\frac{1}{2}$ is a positive constant, $\gamma>0$ is a parameter, $p>2$ and $N \geq 3$.

Equation (1) is derived from a superfluid film equation in plasma physics [11]; see [7-9, 15 ] and the references therein for more physical backgrounds. When $\alpha=1$, the existence of solutions for Eq. (1) was extensively considered in recent years [2, 3, 9, 14-16, 19-21] since the change in $[9,14]$ was introduced. Furthermore, using the change of variables, for general $\alpha>\frac{1}{2}$, the existence of solutions of (1) have been studied; see $[1,4,12]$ and the references therein. Comparing with the semilinear elliptic equations, it is much more challenging and interesting because of the existence of the term $\left(\Delta\left(|u|^{2 \alpha}\right)\right)|u|^{2 \alpha-2} u$. It is worth mentioning that the authors in [20] considered problem (1) with $\alpha=1$. Using the change of variables introduced in [19] and the cut-off function technique in [5], the authors reduced Eq. (1) to a semilinear elliptic equation. Then the existence and boundedness of solution was obtained by the critical point theory when $p \in\left(2,2^{*}\right)$ for $N \geq 4$ or $p \in(2,4)$ for $N=3$. Moreover, they got the asymptotic properties of the solution of (1) by using the arguments in [1,3]. But in [20], what will happen when $p \in[4,6)$ for $N=3$ ?

In this paper, we want to address the existence of Eq. (1) with $\alpha>\frac{1}{2}$ by using the technique of $[5,19,20]$. Furthermore, we can discuss the exponent $p$ from 2 to $2^{*}$ for any $N \geq 3$ by introducing different cut-off functions when $p<4 \alpha$ and $p \geq 4 \alpha$. We also can get the asymptotic properties of the solution of (1) with the use of techniques in $[1,3,20]$.

(c) The Author(s) 2019. This article is distributed under the terms of the Creative Commons Attribution 4.0 International License (http://creativecommons.org/licenses/by/4.0/), which permits unrestricted use, distribution, and reproduction in any medium, provided you give appropriate credit to the original author(s) and the source, provide a link to the Creative Commons license, and indicate if changes were made. 
We assume that the potential function $V$ satisfies $\left(V_{1}\right) 0<V_{0} \leq V(x) \leq \lim _{|x| \rightarrow+\infty} V(x)=$ $V_{\infty}<+\infty$.

Define the space $X=\left\{u \in H^{1}\left(\mathbb{R}^{N}\right): \int_{\mathbb{R}^{N}}|u|^{2(2 \alpha-1)}|\nabla u|^{2} d x<\infty\right\}$. Then, for $u \in X$, the energy functional $I_{\gamma}(u)$ associated with (1) is

$$
I_{\gamma}(u)=\frac{1}{2} \int_{\mathbb{R}^{N}}\left(|\nabla u|^{2}+V(x)|u|^{2}\right) d x+\alpha^{2} \gamma \int_{\mathbb{R}^{N}}|u|^{2(2 \alpha-1)}|\nabla u|^{2} d x-\frac{1}{p} \int_{\mathbb{R}^{N}}|u|^{p} d x
$$

Theorem 1.1 Assume $V(x)=\mu>0$, then Eq. (1) has a positive solution $u_{\gamma}$ satisfying: $(i) u_{\gamma}$ is spherically symmetric and $u_{\gamma}$ decreases with respect to $|x| ;(i i) u_{\gamma} \in C^{2}\left(\mathbb{R}^{N}\right) ;(i i i) u_{\gamma}$ together with its derivatives up to order 2 have exponential decay at infinity $\left|D^{\alpha} u_{\gamma}\right| \leq C e^{-\delta|x|}$, $x \in \mathbb{R}^{N}$, for some $C, \delta>0$ and $|\alpha| \leq 2$. Passing to a subsequence if necessary, it follows that

$$
u_{\gamma} \rightarrow u_{0} \quad \text { in } H^{2}\left(\mathbb{R}^{N}\right) \cap C^{2}\left(\mathbb{R}^{N}\right) \text { as } \gamma \rightarrow 0^{+}
$$

where $u_{0}$ is the ground state of equation $-\Delta u+\mu u=|u|^{p-2} u, x \in \mathbb{R}^{N}$.

Theorem 1.2 Assume that $\left(V_{1}\right)$ holds and $p \in\left(2,2^{*}\right)$. Then there exists a $\gamma_{0}$ such that, for $\gamma \in\left(0, \gamma_{0}\right)$, Eq. (1) has a positive solution $u_{\gamma}$ satisfying $\max _{x \in \mathbb{R}^{N}}\left|\gamma^{\mu} u_{\gamma}(x)\right| \rightarrow 0$ as $\gamma \rightarrow$ $0^{+}$for any $\mu>\frac{1}{2(2 \alpha-1)}$.

Remark 1.1 If $\alpha=1$, the above theorem is essentially Theorem 1.1 of [20]. When $N=3$, $p<4$ is necessary in [20]. But in here, we extend this result to $p<2^{*}$. Moreover, for general $\alpha>\frac{1}{2},[2,15]$ obtain the existence of solutions of (1) for $p \geq 4 \alpha$. But we can obtain the existence of solutions for the case $p<4 \alpha$.

In this paper, we use the following notations: $C$ denotes constant, $\|u\|^{2}=\int_{\mathbb{R}^{N}}\left(|\nabla u|^{2}+\right.$ $\left.u^{2}\right) d x$ for $u \in H^{1}\left(\mathbb{R}^{N}\right),\|u\|_{p}$ denotes the norm of the space $L^{p}\left(\mathbb{R}^{N}\right)$.

\section{The cut-off technique and some lemmas}

We introduce the cut-off function $\zeta(t): \mathbb{R} \rightarrow \mathbb{R}$ such that $\zeta(t)=0$ if $t \leq 0, \zeta(t)=\frac{e^{-\frac{1}{t}}}{e^{-\frac{1}{t}}+e^{-\frac{1}{1-t}}}$ if $0<t<1$ and $\zeta(t)=1$ if $t \geq 1$. The basic property of the function was already used in $[17,18,20]$. It is easy to see that $\zeta(t) \in C^{\infty}(\mathbb{R},[0,1]), 0 \leq \zeta(t) \leq 1$ for all $t \in \mathbb{R}$. Moreover, $\zeta^{\prime}(t)=\frac{\left(2 t^{2}-2 t+1\right) e^{\frac{1-2 t}{t(1-t)}}}{t^{2}(1-t)^{2}\left[1+e^{\frac{1-2 t}{t(1-t)}}\right]^{2}}$ if $0<t<1$ and $\zeta^{\prime}(t)=0$ if $t<0$ or $t>1$. Let $\zeta^{\prime}(0)=\zeta^{\prime}(1)=0$, then $\zeta^{\prime}(t) \geq 0$ is uniformly bounded in $[0,1]$. This means there exists some $C_{0}>0$ such that $\left|\zeta^{\prime}(t)\right| \leq C_{0}$ for any $t \in \mathbb{R}$.

Case I: $4 \alpha>p$. In this case, we assume that

$$
\rho(t)=\zeta^{2}\left[\frac{2^{\frac{1}{2 \alpha-1}}}{2^{\frac{1}{2 \alpha-1}}-1}\left(1-\left(\frac{8 \alpha^{2} \gamma(4 \alpha-p)}{p-2}\right)^{\frac{1}{2(2 \alpha-1)}} t\right)\right]
$$

Then $\rho(t) \in C^{\infty}\left(\mathbb{R}^{+},[0,1]\right)$ and

$$
\rho(t) \begin{cases}=1 & \text { if } 0 \leq t<\left(\frac{p-2}{32 \alpha^{2} \gamma(4 \alpha-p)}\right)^{\frac{1}{2(2 \alpha-1)}}, \\ \in(0,1) & \text { if }\left(\frac{p-2}{32 \alpha^{2} \gamma(4 \alpha-p)}\right)^{\frac{1}{2(2 \alpha-1)}} \leq t<\left(\frac{p-2}{8 \alpha^{2} \gamma(4 \alpha-p)}\right)^{\frac{1}{2(2 \alpha-1)}} \\ =0 & \text { if } t \geq\left(\frac{p-2}{8 \alpha^{2} \gamma(4 \alpha-p)}\right)^{\frac{1}{2(2 \alpha-1)}} .\end{cases}
$$


Moreover, for any $t \in \mathbb{R}^{+}$, we have $0 \geq \rho^{\prime}(t) \geq-\frac{2 \frac{2 \alpha}{2 \alpha-1}}{2 \frac{1}{2 \alpha-1}-1}\left(\frac{8 \alpha^{2} \gamma(4 \alpha-p)}{p-2}\right)^{\frac{1}{2(2 \alpha-1)}} C_{0} \sqrt{\rho(t)}$. Nextly, we assume that $\eta(t)=\rho(-t)$ if $t \leq 0$ and $\eta(t)=\rho(t)$ if $t \geq 0$. It means that

$$
\eta(t) \begin{cases}=\eta(-t) & \text { if } t \leq 0 \\ =1 & \text { if } 0 \leq t<\left(\frac{p-2}{32 \alpha^{2} \gamma(4 \alpha-p)}\right)^{\frac{1}{2(2 \alpha-1)}} \\ \in(0,1) & \text { if }\left(\frac{p-2}{32 \alpha^{2} \gamma(4 \alpha-p)}\right)^{\frac{1}{2(2 \alpha-1)}} \leq t<\left(\frac{p-2}{8 \alpha^{2} \gamma(4 \alpha-p)}\right)^{\frac{1}{2(2 \alpha-1)}} \\ =0 & \text { if } t \geq\left(\frac{p-2}{8 \alpha^{2} \gamma(4 \alpha-p)}\right)^{\frac{1}{2(2 \alpha-1)}}\end{cases}
$$

$\eta(t) \in C_{0}^{\infty}(\mathbb{R},[0,1])$ and $\eta^{\prime}(t) t \leq 0$ for $t \in \mathbb{R}^{+}$. Furthermore, for $t \in \mathbb{R}^{+}$, we have

$$
t \eta^{\prime}(t) \geq \begin{cases}-\frac{1}{2 \frac{1}{2 \alpha-1}-1} C_{0} & \text { if } 0 \leq t<\left(\frac{p-2}{32 \alpha^{2} \gamma(4 \alpha-p)}\right)^{\frac{1}{2(2 \alpha-1)}} \\ -\frac{2 \frac{1}{2 \alpha-1}}{2 \frac{1}{2 \alpha-1}-1} C_{0} \sqrt{\eta(t)} & \text { if }\left(\frac{p-2}{32 \alpha^{2} \gamma(4 \alpha-p)}\right)^{\frac{1}{2(2 \alpha-1)}} \leq t<\left(\frac{p-2}{8 \alpha^{2} \gamma(4 \alpha-p)}\right)^{\frac{1}{2(2 \alpha-1)}} \\ 0 & \text { if } t \geq\left(\frac{p-2}{8 \alpha^{2} \gamma(4 \alpha-p)}\right)^{\frac{1}{2(2 \alpha-1)}}\end{cases}
$$

Case II: $p \geq 4 \alpha$. In this case, we let

$$
\rho(t)=\zeta^{2}\left[\frac{2^{\frac{1}{2 \alpha-1}}}{2^{\frac{1}{2 \alpha-1}}-1}\left(1-\left(\frac{8 \alpha^{2} \gamma(6-p)}{p-2}\right)^{\frac{1}{2(2 \alpha-1)}} t\right)\right] .
$$

Similar to the case I, we assume that $\eta(t)=\rho(-t)$ if $t \leq 0$ and $\eta(t)=\rho(t)$ if $t \geq 0$. Then $0 \geq \rho^{\prime}(t) \geq-2 \frac{2 \frac{1}{2 \alpha-1}}{2 \frac{1}{2 \alpha-1}-1}\left(\frac{8 \alpha^{2} \gamma(6-p)}{p-2}\right)^{\frac{1}{2(2 \alpha-1)}} C_{0} \sqrt{\rho(t)}$ and

$$
\eta(t) \begin{cases}=\eta(-t) & \text { if } t \leq 0 \\ =1 & \text { if } 0 \leq t<\left(\frac{p-2}{32 \alpha^{2} \gamma(6-p)}\right)^{\frac{1}{2(2 \alpha-1)}} \\ \in(0,1) & \text { if }\left(\frac{p-2}{32 \alpha^{2} \gamma(6-p)}\right)^{\frac{1}{2(2 \alpha-1)}} \leq t<\left(\frac{p-2}{8 \alpha^{2} \gamma(6-p)}\right)^{\frac{1}{2(2 \alpha-1)}} \\ =0 & \text { if } t \geq\left(\frac{p-2}{8 \alpha^{2} \gamma(6-p)}\right)^{\frac{1}{2(2 \alpha-1)}}\end{cases}
$$

For $p \in\left(2,2^{*}\right)$, we construct an auxiliary function $g_{\gamma}(t): \mathbb{R} \rightarrow \mathbb{R}^{+}$just like:

$$
g_{\gamma}(t)=\sqrt{\left(\frac{1}{2}+2 \alpha^{2} \gamma|t|^{2(2 \alpha-1)}\right) \eta(t)+\frac{1}{2}}
$$

where $\eta(t)$ take the form (3) if $p<4 \alpha$ and the form (4) if $p \geq 4 \alpha$. Then we know that $g_{\gamma}(0)=1, \frac{\sqrt{2}}{2} \leq g_{\gamma}(t) \leq \sqrt{\frac{14-3 p}{4(4-p)}}$ if $p \leq 4 \alpha, \frac{\sqrt{2}}{2} \leq g_{\gamma}(t) \leq \sqrt{\frac{22-3 p}{4(6-p)}}$ if $p \geq 4 \alpha$,

$$
g_{\gamma}^{\prime}(t) t=\frac{\left(\frac{1}{2}+2 \alpha^{2} \gamma|t|^{2(2 \alpha-1)}\right) \eta^{\prime}(t) t+4(2 \alpha-1) \gamma|t|^{2(2 \alpha-1) \eta(t)}}{2\left[\left(\frac{1}{2}+2 \alpha^{2} \gamma|t|^{2(2 \alpha-1)}\right) \eta(t)+\frac{1}{2}\right]^{\frac{1}{2}}}
$$

and $g_{\gamma}^{\prime}(t) t=-g_{\gamma}^{\prime}(-t) t$. Define $G_{\gamma}(t)=\int_{0}^{t} g_{\gamma}(s) d s$. Then the inverse function $G_{\gamma}^{-1}(t)$ exists and is an odd function. Furthermore, $G_{\gamma}, G_{\gamma}^{-1} \in C^{\infty}(\mathbb{R}, \mathbb{R})$.

Lemma 2.1 The following properties hold:

$$
\lim _{t \rightarrow 0} \frac{G_{\gamma}^{-1}(t)}{t}=1 ; \quad \lim _{t \rightarrow \infty} \frac{G_{\gamma}^{-1}(t)}{t}=\sqrt{2}
$$




$$
\begin{aligned}
& \sqrt{\frac{4(4 \alpha-p)}{16 \alpha-2-3 p}}|t| \leq\left|G_{\gamma}^{-1}(t)\right| \leq \sqrt{2}|t|, \quad \text { for all } t \in \mathbb{R} \text { and } p \leq 4 \alpha \\
& \sqrt{\frac{4(6-p)}{22-3 p}}|t| \leq\left|G_{\gamma}^{-1}(t)\right| \leq \sqrt{2}|t|, \quad \text { for all } t \in \mathbb{R} \text { and } p \geq 4 \alpha \\
& -C \leq \frac{g_{\gamma}^{\prime}(t) t}{g_{\gamma}(t)} \leq \frac{(8 \alpha-2-p)(p-2)}{16 \alpha-2-3 p}, \quad \text { for all } t \in \mathbb{R} \text { and } p \leq 4 \alpha \\
& -C \leq \frac{g_{\gamma}^{\prime}(t) t}{g_{\gamma}(t)} \leq \frac{(6-p)(p-2)}{14-3 p}, \quad \text { for all } t \in \mathbb{R} \text { and } p \geq 4 \alpha
\end{aligned}
$$

Proof The proofs of (6)-(8) are similar to those of Lemma 2.1 in [20], so we omit them. For the case (9), By the definition of $g_{\gamma}$ and (3), we obtain

$$
\frac{g_{\gamma}^{\prime}(t) t}{g_{\gamma}(t)} \geq \frac{-C\left(\frac{1}{2}+2 \alpha^{2} \gamma t^{2(2 \alpha-1)}\right) \sqrt{\eta(t)}}{\left(1+4 \alpha^{2} \gamma t^{2(2 \alpha-1)}\right) \eta(t)+1} \geq \begin{cases}-C & \text { if } 0 \leq t<\left(\frac{p-2}{8 \alpha^{2} \gamma(4 \alpha-p)}\right)^{\frac{1}{2(2 \alpha-1)}} \\ 0 & \text { if } t \geq\left(\frac{p-2}{8 \alpha^{2} \gamma(4 \alpha-p)}\right)^{\frac{1}{2(2 \alpha-1)}}\end{cases}
$$

Moreover, for $0 \leq t<\left(\frac{p-2}{8 \alpha^{2} \gamma(4 \alpha-p)}\right)^{\frac{1}{2(2 \alpha-1)}}$, we know that $(p-2)+(4 p-16 \alpha) \alpha^{2} \gamma t^{2(2 \alpha-1)} \geq$ $\frac{p-2}{2}>0$. Hence

$$
\begin{aligned}
& \frac{p-2}{2}-\frac{g_{\gamma}^{\prime}(t) t}{g_{\gamma}(t)} \\
& \quad=\frac{\left[(p-2)+(4 p-16 \alpha) \alpha^{2} \gamma t^{2(2 \alpha-1)}\right] \eta(t)-\eta^{\prime}(t) t\left(1+4 \alpha^{2} \gamma t^{2(2 \alpha-1)}\right)+p-2}{4 g_{\gamma}^{2}(t)} \\
& \quad \geq \frac{p-2}{2\left[\left(1+4 \alpha^{2} \gamma t^{2(2 \alpha-1)}\right) \eta(t)+1\right]} \geq \frac{(p-2)(4 \alpha-p)}{16 \alpha-2-3 p},
\end{aligned}
$$

which yields the result.

For the case (10), since $p \geq 4 \alpha$, it is easy to see that $(p-2)+(4 p-16 \alpha) \alpha^{2} \gamma t^{2(2 \alpha-1)}>0$. Then

$$
\frac{p-2}{2}-\frac{g_{\gamma}^{\prime}(t) t}{g_{\gamma}(t)} \geq \frac{p-2}{2\left[\left(1+4 \alpha^{2} \gamma t^{2(2 \alpha-1)}\right) \eta(t)+1\right]} \geq \frac{(p-2)(6-p)}{22-3 p} .
$$

According to the properties of $g_{\gamma}$, we will focus on the existence of positive solutions for the following general quasilinear Schrödinger equation:

$$
-\operatorname{div}\left(g_{\gamma}^{2}(u) \nabla u\right)+g_{\gamma}(u) g_{\gamma}^{\prime}(u)|\nabla u|^{2}+V(x) u=|u|^{p-2} u, \quad x \in \mathbb{R}^{N} .
$$

The energy functional of (11) is

$$
E_{\gamma}(u)=\frac{1}{2} \int_{\mathbb{R}^{N}} g_{\gamma}^{2}(u)|\nabla u|^{2} d x+\frac{1}{2} \int_{\mathbb{R}^{N}} V(x) u^{2} d x-\frac{1}{p} \int_{\mathbb{R}^{N}}|u|^{p} d x .
$$

Furthermore, we introduce $G_{\gamma}(t)=\int_{0}^{t} g_{\gamma}(s) d s$ and the change of variables $u=G_{\gamma}^{-1}(v)$. Then that functional $E_{\gamma}$ can be rewritten as

$$
J_{\gamma}(v)=\frac{1}{2} \int_{\mathbb{R}^{N}}|\nabla v|^{2} d x+\frac{1}{2} \int_{\mathbb{R}^{N}} V(x)\left|G_{\gamma}^{-1}(v)\right|^{2} d x-\frac{1}{p} \int_{\mathbb{R}^{N}}\left|G_{\gamma}^{-1}(v)\right|^{p} d x
$$


This means that the function $v$ is the solution of the following equation:

$$
-\Delta v+V(x) \frac{G_{\gamma}^{-1}(v)}{g_{\gamma}\left(G_{\gamma}^{-1}(v)\right)}-\frac{\left|G_{\gamma}^{-1}(v)\right|^{p-2} G_{\gamma}^{-1}(v)}{g_{\gamma}\left(G_{\gamma}^{-1}(v)\right)}=0, \quad x \in \mathbb{R}^{N}
$$

From Lemma 2.1, $J_{\gamma}$ is well defined in $H^{1}\left(\mathbb{R}^{N}\right)$ and of class $C^{1}$.

Lemma 2.2 Assume that $V(x)=\mu>0$ and $h(v)=\frac{\left|G_{\gamma}^{-1}(v)\right|^{p-2} G_{\gamma}^{-1}(v)}{g_{\gamma}\left(G_{\gamma}^{-1}(v)\right)}-\mu \frac{G_{\gamma}^{-1}(v)}{g_{\gamma}\left(G_{\gamma}^{-1}(v)\right)}$. Then

$$
\lim _{v \rightarrow 0} \frac{h(v)}{v}=-\mu, \quad \lim _{v \rightarrow \infty} \frac{h(v)}{v^{\frac{N+2}{N-2}}}=0
$$

and there is a $\xi>0$ such that $H(\xi)=\int_{0}^{\xi} h(s) d s>0$.

Proof From Lemma 2.1, we have $G_{\gamma}^{-1}(v) \rightarrow 0$ and $g_{\gamma}\left(G_{\gamma}^{-1}(v)\right) \rightarrow 1$ as $v \rightarrow 0$. $G_{\gamma}^{-1}(v) \rightarrow \infty$ and $g_{\gamma}\left(G_{\gamma}^{-1}(v)\right) \rightarrow \frac{1}{\sqrt{2}}$ as $v \rightarrow \infty$. Hence

$$
\begin{aligned}
& \lim _{v \rightarrow 0} \frac{h(v)}{v}=\lim _{v \rightarrow 0} \frac{\left|G_{\gamma}^{-1}(v)\right|^{p-2} G_{\gamma}^{-1}(v)}{v g_{\gamma}\left(G_{\gamma}^{-1}(v)\right)}-\mu \lim _{\nu \rightarrow 0} \frac{G_{\gamma}^{-1}(v)}{v g_{\gamma}\left(G_{\gamma}^{-1}(v)\right)}=-\mu, \\
& \lim _{v \rightarrow \infty} \frac{h(v)}{v^{N+2}}=\lim _{v \rightarrow \infty} \frac{\left|G_{\gamma}^{-1}(v)\right|^{p-2} G_{\gamma}^{-1}(v)}{G_{\gamma}^{-1}(v)^{\frac{N+2}{N-2}}} \frac{G_{\gamma}^{-1}(v)^{\frac{N+2}{N-2}}}{v^{\frac{N+2}{N-2}} g_{\gamma}\left(G_{\gamma}^{-1}(v)\right)}-0=0 .
\end{aligned}
$$

Moreover,

$$
\begin{aligned}
\int_{0}^{G_{\gamma}(\xi)} h(s) d s & =\int_{0}^{G_{\gamma}(\xi)}\left|G_{\gamma}^{-1}(s)\right|^{p-2} G_{\gamma}^{-1}(s) d G_{\gamma}^{-1}(s)-\mu \int_{0}^{G_{\gamma}(\xi)} G_{\gamma}^{-1}(s) d G_{\gamma}^{-1}(s) \\
& =\frac{\xi^{p}}{p}-\frac{\mu \xi}{2} .
\end{aligned}
$$

Hence, there is a $\xi>0$ such that $H(\xi)=\int_{0}^{\xi} h(s) d s>0$.

Lemma 2.3 Assume that $\left(V_{1}\right)$ holds. Then any $(P S)$ sequence $\left\{v_{n}\right\}$ of $J_{\gamma}$ is bounded.

Proof Let $\left\{v_{n}\right\}$ be a $(P S)$ sequence, we have

$$
\begin{aligned}
J_{\gamma}\left(v_{n}\right)= & \frac{1}{2} \int_{\mathbb{R}^{N}}\left|\nabla v_{n}\right|^{2} d x+\frac{1}{2} \int_{\mathbb{R}^{N}} V(x)\left|G_{\gamma}^{-1}\left(v_{n}\right)\right|^{2} d x-\frac{1}{p} \int_{\mathbb{R}^{N}}\left|G_{\gamma}^{-1}\left(v_{n}\right)\right|^{p} d x \\
= & c_{\gamma}+o_{n}(1), \\
\left\langle J_{\gamma}^{\prime}\left(v_{n}\right), \psi\right\rangle= & \int_{\mathbb{R}^{N}} \nabla v_{n} \nabla \psi d x+\int_{\mathbb{R}^{N}} V(x) \frac{G_{\gamma}^{-1}\left(v_{n}\right)}{g_{\gamma}\left(G_{\gamma}^{-1}\left(v_{n}\right)\right)} \psi d x \\
& -\int_{\mathbb{R}^{N}} \frac{\left|G_{\gamma}^{-1}\left(v_{n}\right)\right|^{p-2} G_{\gamma}^{-1}\left(v_{n}\right)}{g_{\gamma}\left(G_{\gamma}^{-1}\left(v_{n}\right)\right)} \psi d x=o(\|\psi\|)
\end{aligned}
$$

for all $\psi \in H^{1}\left(\mathbb{R}^{N}\right)$. 
Taking $\psi_{n}=G_{\gamma}^{-1}\left(v_{n}\right) g_{\gamma}\left(G_{\gamma}^{-1}\left(v_{n}\right)\right)$. From Lemma 2.1, we can get

$$
\left|\nabla \psi_{n}\right|=\left|\left[1+\frac{G_{\gamma}^{-1}\left(v_{n}\right) g_{\gamma}^{\prime}\left(G_{\gamma}^{-1}\left(v_{n}\right)\right)}{g_{\gamma}\left(G_{\gamma}^{-1}\left(v_{n}\right)\right)}\right] \nabla v_{n}\right| \leq C_{0}\left|\nabla v_{n}\right|
$$

and $\left|\psi_{n}\right| \leq \sqrt{\frac{16 \alpha-2-3 p}{2(4 \alpha-p)}}\left|v_{n}\right|$ if $p \leq 4 \alpha,\left|\psi_{n}\right| \leq \sqrt{\frac{22-3 p}{2(6-p)}}\left|v_{n}\right|$ if $p \geq 4 \alpha$.

If $p \leq 4 \alpha$, combining (13), (14) and (9) of Lemma 2.1, we get

$$
\begin{aligned}
p c_{\gamma}+o(1)+o(1)\left\|v_{n}\right\| & \geq \frac{(p-2)(4 \alpha-p)}{16 \alpha-2-3 p} \int_{\mathbb{R}^{N}}\left|\nabla v_{n}\right|^{2} d x+\frac{p-2}{2} \int_{\mathbb{R}^{N}} V(x)\left|G_{\gamma}^{-1}\left(v_{n}\right)\right|^{2} d x \\
& \geq \frac{(p-2)(4 \alpha-p)}{16 \alpha-2-3 p}\|v\|^{2} .
\end{aligned}
$$

If $p \geq 4 \alpha$, combining (13), (14) and (10) of Lemma 2.1, we get

$$
\begin{aligned}
p c_{\gamma}+o(1)+o(1)\left\|v_{n}\right\| & \geq \frac{(p-2)(6-p)}{22-3 p} \int_{\mathbb{R}^{N}}\left|\nabla v_{n}\right|^{2} d x+\frac{p-2}{2} \int_{\mathbb{R}^{N}} V(x)\left|G_{\gamma}^{-1}\left(v_{n}\right)\right|^{2} d x \\
& \geq \frac{(p-2)(6-p)}{22-3 p}\|v\|^{2} .
\end{aligned}
$$

This shows the boundedness of $\left\{v_{n}\right\}$ in $H^{1}\left(\mathbb{R}^{N}\right)$.

\section{The proof of theorems}

Proof of Theorem 1.1 If $V(x)=\mu>0$, from Lemma 2.2, a standard method similar to the proof of [6] indicates that there is a solution $v_{\gamma}$ of Eq. (12) satisfies: (i) $v_{\gamma}>0$ is spherically symmetric and $v_{\gamma}$ decrease with respect to $|x|$; (ii) $v_{\gamma} \in C^{2}\left(\mathbb{R}^{N}\right)$; (iii) $v_{\gamma}$ together with its derivatives up to order 2 have exponential decay at infinity: $\left|D^{\alpha} v_{\gamma}\right| \leq C e^{-\delta|x|}, x \in \mathbb{R}^{N}$, for some $C, \delta>0$ and $|\alpha| \leq 2$. Then, according the techniques of $[2,10,20]$, we can deduce that $u_{\gamma}=G^{-1}\left(v_{\gamma}\right)$ is a solution of problem (1) and $\left\|\nabla u_{\gamma}\right\|_{\infty} \leq C$. Moreover, there is a $u_{0}$, such that $u_{\gamma}=G^{-1}\left(v_{\gamma}\right) \rightarrow u_{0}$, where $u_{0}$ is a nonnegative solution of problem $-\Delta u+\mu u=|u|^{p-2} u$ in $\mathbb{R}^{N}$. Furthermore, similar to the proof of Lemma 4.5 in [20], we can deduce that $u_{\gamma} \rightarrow u_{0}$ in $H^{2}\left(\mathbb{R}^{N}\right)$.

Similar to the proof of Lemma 5.5 in [3] or Lemma 4.6 in [20], we know that $\left|v_{\gamma}\right| \leq$ $\frac{C}{|x|}\left\|v_{\gamma}\right\| \leq \frac{C}{|x|},|x| \geq 1$. Then, for any $\varepsilon>0$ and $q>2$, there exists $R>0$ independent of $\gamma$, such that

$$
\begin{aligned}
& \left\|-\mu \frac{G_{\gamma}^{-1}\left(v_{\gamma}\right)}{g_{\gamma}\left(G_{\gamma}^{-1}\left(v_{\gamma}\right)\right)}+\frac{\left|G_{\gamma}^{-1}\left(v_{\gamma}\right)\right|^{p-2} G_{\gamma}^{-1}\left(v_{\gamma}\right)}{g_{\gamma}\left(G_{\gamma}^{-1}\left(v_{\gamma}\right)\right)}\right\|_{L^{q}\left(\mathbb{R}^{N} \backslash B_{R}(0)\right)}<\varepsilon, \\
& \left\|\mu u_{0}\right\|_{L^{q}\left(\mathbb{R}^{N} \backslash B_{R}(0)\right)}+\left\|\left|u_{0}\right|^{p-2} u_{0}\right\|_{L^{q}\left(\mathbb{R}^{N} \backslash B_{R}(0)\right)}<\varepsilon .
\end{aligned}
$$

From $\left\|u_{\gamma}\right\|_{\infty}=\left\|G_{\gamma}^{-1}\left(v_{\gamma}\right)\right\|_{\infty} \leq C$, we get $G_{\gamma}^{-1}\left(v_{\gamma}\right) \rightarrow u_{0}$, a.e. in $\mathbb{R}^{N}$ and

$$
-\mu \frac{G_{\gamma}^{-1}\left(v_{\gamma}\right)}{\sqrt{1+2 \alpha^{2} \gamma\left|G_{\gamma}^{-1}\left(v_{\gamma}\right)\right|^{2(2 \alpha-1)}}} \rightarrow-\mu u_{0}, \quad \text { a.e. in } \mathbb{R}^{N} \text {. }
$$


Using the Lebesgue dominated convergence theorem, we have

$$
\left\|-\mu \frac{G_{\gamma}^{-1}\left(v_{\gamma}\right)}{\sqrt{1+2 \alpha^{2} \gamma\left|G_{\gamma}^{-1}\left(v_{\gamma}\right)\right|^{2(2 \alpha-1)}}}-\mu u_{0}\right\|_{L^{q}\left(B_{R}(0)\right)}+\left\|\left|u_{\gamma}\right|^{p-2} u_{\gamma}-\left|u_{0}\right|^{p-2} u_{0}\right\|_{L^{q}\left(B_{R}(0)\right)} \rightarrow 0 .
$$

Hence $\lim \sup _{\gamma \rightarrow 0^{+}}\left\|\Delta\left(v_{\gamma}-u_{0}\right)\right\|_{L^{q}} \leq 2 \varepsilon$. From the arbitrariness of $\varepsilon$, we have $v_{\gamma} \rightarrow u_{0}$ in $W^{2, q}\left(\mathbb{R}^{N}\right)$ for any $q>2$ as $\gamma \rightarrow 0^{+}$. From the Sobolev embedding, we get $v_{\gamma} \rightarrow u_{0}$ in $C^{1, \alpha}\left(\mathbb{R}^{N}\right)$. Moreover, the bootstrap arguments indicate that $v_{\gamma} \rightarrow u_{0}$ in $C^{2}\left(\mathbb{R}^{N}\right)$.

From the definition of $v_{\gamma}$, we have

$$
\left|v_{\gamma}-u_{\gamma}\right|=\left|\int_{0}^{u_{\gamma}}\left(\sqrt{1+2 \alpha^{2} \gamma|t|^{2(2 \alpha-1)}}-1\right) d t\right| \leq \frac{\alpha^{2} \gamma u_{\gamma}^{4 \alpha-1}}{4 \alpha-1}
$$

Hence $\sup _{x \in \mathbb{R}^{N}}\left|v_{\gamma}(x)-u_{\gamma}(x)\right| \leq C \gamma\left\|u_{\gamma}\right\|_{\infty}^{3} \rightarrow 0$ as $\gamma \rightarrow 0$.

Furthermore, from the definition of $v_{\gamma}$, we know that $\nabla v_{\gamma}=g_{\gamma}\left(u_{\gamma}\right) \nabla u_{\gamma}$ and

$$
\begin{aligned}
& \sup _{x \in \mathbb{R}^{N}}\left|\nabla v_{\gamma}(x)-\nabla u_{\gamma}(x)\right| \\
& =\sup _{x \in \mathbb{R}^{N}}\left|\left(g_{\gamma}\left(u_{\gamma}\right)-1\right) \nabla u_{\gamma}\right|=\sup _{x \in \mathbb{R}^{N}}\left|\frac{2 \alpha^{2} \gamma u_{\gamma}^{2(2 \alpha-1)} \nabla u_{\gamma}}{\sqrt{1+2 \alpha^{2} \gamma u_{\gamma}^{2(2 \alpha-1)}}+1}\right| \\
& \leq \sup _{x \in \mathbb{R}^{N}}\left|\alpha^{2} \gamma u_{\gamma}^{2(2 \alpha-1)} \nabla u_{\gamma}\right| \leq \alpha^{2} \gamma|| u_{\gamma}||_{\infty}^{2(2 \alpha-1)}|| \nabla u_{\gamma}||_{\infty} \rightarrow 0, \\
& \left.\sup _{x \in \mathbb{R}^{N}}\left|-\mu \frac{G_{\gamma}^{-1}\left(v_{\gamma}\right)}{g_{\gamma}\left(G_{\gamma}^{-1}\left(v_{\gamma}\right)\right)}+\frac{\left|G_{\gamma}^{-1}\left(\nu_{\gamma}\right)\right|^{p-2} G_{\gamma}^{-1}\left(v_{\gamma}\right)}{g_{\gamma}\left(G_{\gamma}^{-1}\left(v_{\gamma}\right)\right)}-\mu u_{\gamma}-\right| u_{\gamma}\right|^{p-2} u_{\gamma} \mid \rightarrow 0
\end{aligned}
$$

as $\gamma \rightarrow 0$. On the other hand,

$$
\left|\Delta u_{\gamma}\right|=\left|\frac{1}{1+2 \alpha^{2} \gamma\left|u_{\gamma}\right|^{2(2 \alpha-1)}}\left[2(2 \alpha-1) \alpha^{2} \gamma\left|u_{\gamma}\right|^{4 \alpha-4} u_{\gamma}\left|\nabla u_{\gamma}\right|^{2}-\mu u_{\gamma}+\left|u_{\gamma}\right|^{p-2} u_{\gamma}\right]\right| \leq C .
$$

It indicates that

$$
\begin{aligned}
& \sup _{x \in \mathbb{R}^{N}}\left|\Delta\left(v_{\gamma}-u_{\gamma}\right)\right| \\
& \leq \sup _{x \in \mathbb{R}^{N}}\left|2 \alpha^{2} \gamma u_{\gamma}^{2(2 \alpha-1)} \Delta u_{\gamma}\right|+\left.\sup _{x \in \mathbb{R}^{N}}\left|2(2 \alpha-1) \alpha^{2} \gamma u_{\gamma}^{4 \alpha-3}\right| \nabla u_{\gamma}\right|^{2} \mid \\
& \quad+\left.\sup _{x \in \mathbb{R}^{N}}\left|-\mu \frac{G_{\gamma}^{-1}\left(v_{\gamma}\right)}{g_{\gamma}\left(G_{\gamma}^{-1}\left(v_{\gamma}\right)\right)}+\frac{\left|G_{\gamma}^{-1}\left(v_{\gamma}\right)\right|^{p-2} G_{\gamma}^{-1}\left(v_{\gamma}\right)}{g_{\gamma}\left(G_{\gamma}^{-1}\left(v_{\gamma}\right)\right)}-\mu u_{\gamma}-\right| u_{\gamma}\right|^{p-2} u_{\gamma} \mid \rightarrow 0 .
\end{aligned}
$$

As in [3] Lemma 5.5, or [20] Lemma 4.6, (15) together with the Sobolev interpolation inequality yields

$$
\sup _{x \in \mathbb{R}^{N}}\left|D^{j}\left(v_{\gamma}-u_{\gamma}\right)\right| \rightarrow 0, \quad|j| \leq 2 .
$$

Multiplying $u_{\gamma}$ by (1), we have

$$
\int_{x \in \mathbb{R}^{N}}\left(1+4 \alpha^{3} \gamma u_{\gamma}^{2}\right)\left|\nabla u_{\gamma}\right|^{2}+\mu u_{\gamma}^{2}-u_{\gamma}^{p} d x=0
$$


This implies that

$$
\int_{x \in \mathbb{R}^{N}} \mu u_{\gamma}^{2}-u_{\gamma}^{p}<0
$$

If $u_{\gamma}(0)=\left\|u_{\gamma}\right\|_{L^{\infty}} \leq \mu^{\frac{1}{p-2}}$, one has $\mu u_{\gamma}^{2}-u_{\gamma}^{p} \geq 0$, from which we arrive at a contradiction. Then we get $u_{\gamma}(0)>\mu^{\frac{1}{p-2}}$. Since $u_{\gamma} \rightarrow u_{0}$ in $C^{2}$, we can obtain $u_{0}(0) \geq \mu^{\frac{1}{p-2}}$. By the maximum principle, we finally get $u_{0}>0$.

The proof of Theorem 1.2 From Lemma 2.1, a standard discussion shows that $J_{\gamma}$ satisfies the mountain pass geometric hypothesis. Hence, there exists a $(P S)$ sequence $\left\{v_{n}\right\} \subset$ $H^{1}\left(\mathbb{R}^{N}\right)$, such that $J_{\gamma}\left(v_{n}\right) \rightarrow c_{\gamma}$ and $J_{\gamma}^{\prime}\left(v_{n}\right) \rightarrow 0$, where $c_{\gamma}=\inf _{\xi \in \Gamma_{\gamma}} \sup _{t \in[0,1]} J_{\gamma}(\xi(t)), \Gamma_{\gamma}=$ $\left\{\xi(t) \in C\left([0,1], H^{1}\left(\mathbb{R}^{N}\right)\right): \xi(0)=0, \xi(1) \neq 0, J_{\gamma}(\xi(1))<0\right\}$. Then, from Lemma 2.3 , we see that the sequence $\left\{v_{n}\right\}$ is bounded. This indicates that there is a subsequence of $\left\{v_{n}\right\}$, denoted still by $\left\{v_{n}\right\}$, there is $v_{\gamma} \in H^{1}\left(\mathbb{R}^{N}\right)$ such that $v_{n} \rightarrow v_{\gamma}$ in $H^{1}\left(\mathbb{R}^{N}\right), v_{n} \rightarrow v_{\gamma}$ in $L_{\text {loc }}^{q}\left(\mathbb{R}^{N}\right), q \in\left[2,2^{*}\right)$. Hence, using Lebesgue dominated convergence theorem, it is easy to see that $J_{\gamma}^{\prime}\left(v_{\gamma}\right)=0$. Furthermore, we can replace $v_{n}$ by $\left|v_{n}\right|$. Hence, we can assume that $v_{n} \geq 0$ in $\mathbb{R}^{N}$ and $v_{\gamma} \geq 0$. If $v_{\gamma} \neq 0$, then $v_{\gamma}$ is a positive solution of Eq. (12). By contradiction, we assume that $v_{\gamma}=0$. In this time, consider the functional $J_{\gamma}^{\infty}: H^{1}\left(\mathbb{R}^{N}\right) \rightarrow \mathbb{R}$ by

$$
J_{\gamma}^{\infty}=\frac{1}{2} \int_{\mathbb{R}^{N}}\left(\left|\nabla v_{n}\right|^{2}+V_{\infty}\left|G_{\gamma}^{-1}\left(v_{n}\right)\right|^{2}\right) d x-\frac{1}{p} \int_{\mathbb{R}^{N}}\left|G_{\gamma}^{-1}\left(v_{n}\right)\right|^{p} d x
$$

Then we get a contradiction as in a similar proof to $[9,19,20]$ by using the compactness lemma [13]. Hence, $v_{\gamma}$ is a nontrivial solution of Eq. (12). By using the fact that $G_{\gamma}^{-1}(t) \in C^{2}$ together with Lemma 2.1, a direct computation shows that $u=G_{\gamma}^{-1}(v) \in C^{2}\left(\mathbb{R}^{N}\right) \cap H^{1}\left(\mathbb{R}^{N}\right)$. If $v_{\gamma}$ is a critical point for $J_{\gamma}$, we know that

$$
\begin{aligned}
& \int_{\mathbb{R}^{N}}\left[\nabla v \nabla \psi+V(x) \frac{G_{\gamma}^{-1}(v)}{g_{\gamma}\left(G_{\gamma}^{-1}(v)\right)} \psi-\frac{\left|G_{\gamma}^{-1}(v)\right|^{p-2} G_{\gamma}^{-1}(v)}{g_{\gamma}\left(G_{\gamma}^{-1}(v)\right)} \psi\right] d x=0 \\
& \text { for all } \psi \in H^{1}\left(\mathbb{R}^{N}\right) .
\end{aligned}
$$

Taking $\psi=g_{\gamma}(u) \varphi \in C_{0}^{2}\left(\mathbb{R}^{N}\right) \subset H^{1}\left(\mathbb{R}^{N}\right)$ in $(16)$, we have

$$
\int_{\mathbb{R}^{N}}\left[g_{\gamma}^{2}(u) \nabla u \nabla \varphi+g_{\gamma}(u) g_{\gamma}^{\prime}(u)|\nabla u|^{2} \varphi+V(x) u \varphi+|u|^{p-2} u \varphi\right] d x=0 .
$$

It means that $u$ is a classical solution of (11). In the next part of this section, we will prove that $u=G^{-1}\left(v_{\gamma}\right)$ is the solution of Eq. (1).

If $p \leq 4 \alpha$, we define the functional $P: H^{1}\left(\mathbb{R}^{N}\right) \rightarrow \mathbb{R}$ by

$$
P(v)=\frac{1}{2} \int_{\mathbb{R}^{N}}|\nabla v|^{2} d x+2 V_{\infty} \int_{\mathbb{R}^{N}}|v|^{2} d x-\frac{1}{p}\left[\frac{4(4 \alpha-p)}{16-2 \alpha-3 p}\right]^{\frac{p}{2}} \int_{\mathbb{R}^{N}}|v|^{p} d x
$$

Then the function $v$ satisfies the equation

$$
-\Delta v+4 V_{\infty} v=\left[\frac{4(4 \alpha-p)}{16-2 \alpha-3 p}\right]^{\frac{p}{2}}|v|^{p-2} v, \quad x \in \mathbb{R}^{N}
$$


From Jeanjean and Tanaka [10], if we consider the set $\Gamma=\left\{\xi \in C\left([0,1], H^{1}\left(\mathbb{R}^{N}\right)\right): \xi(0)=\right.$ $0, \xi(1) \neq 0, P(\xi(1))<0\}$. Then $m=\inf _{\xi \in \Gamma} \sup _{t \in[0,1]} P(\xi(t))$ is the least energy level of the functional $P(v)$.

Since $v_{\gamma}$ is a critical point of $J_{\gamma}$, one has

$$
p c_{\gamma}=p J_{\gamma}\left(v_{\gamma}\right)-\left\langle J_{\gamma}^{\prime}\left(v_{\gamma}\right), G_{\gamma}^{-1}\left(v_{\gamma}\right) g_{\gamma}\left(G_{\gamma}^{-1}\left(v_{\gamma}\right)\right)\right\rangle \geq \frac{(p-2)(4 \alpha-p)}{16-2 \alpha-3 p} \int_{\mathbb{R}^{N}}\left|\nabla v_{\gamma}\right|^{2} d x
$$

This indicates that

$$
\left\|\nabla v_{\gamma}\right\|_{2}^{2} \leq \frac{p(16-2 \alpha-3 p)}{(p-2)(4 \alpha-p)} c_{\gamma}
$$

Furthermore, from the property (7) of Lemma 2.1, we can deduce that $J_{\gamma}(v) \leq P(v)$ and $\Gamma \subset \Gamma_{\gamma}$. Hence

$$
c_{\gamma}=\inf _{\xi \in \Gamma_{\gamma}} \sup _{t \in[0,1]} J_{\gamma}(\xi(t)) \leq \inf _{\xi \in \Gamma} \sup _{t \in[0,1]} J_{\gamma}(\xi(t)) \leq \inf _{\xi \in \Gamma} \sup _{t \in[0,1]} P(\xi(t)):=m
$$

and

$$
\left\|\nabla v_{\gamma}\right\|_{2}^{2} \leq \frac{p(16-2 \alpha-3 p)}{(p-2)(4 \alpha-p)} m
$$

Using the Sobolev inequality, we can get

$$
\left\|v_{\gamma}\right\|_{2^{*}} \leq \sqrt{\frac{p m(16-2 \alpha-3 p)}{S(p-2)(4 \alpha-p)}}
$$

where $\mathrm{S}$ is the best Sobolev constant.

If $p \geq 4 \alpha$, we define the function $P: H^{1}\left(\mathbb{R}^{N}\right) \rightarrow \mathbb{R}$ by

$$
P(v)=\frac{1}{2} \int_{\mathbb{R}^{N}}|\nabla v|^{2} d x+2 V_{\infty} \int_{\mathbb{R}^{N}}|v|^{2} d x-\frac{1}{p}\left[\frac{4(6-p)}{22-3 p}\right]^{\frac{p}{2}} \int_{\mathbb{R}^{N}}|v|^{p} d x,
$$

the set $\Gamma$ and $m$ are defined like $p \leq 4 \alpha$. In this time, if $v_{\gamma}$ is a critical point of $J_{\gamma}$,

$$
p c_{\gamma}=p J_{\gamma}\left(v_{\gamma}\right)-\left\langle J_{\gamma}^{\prime}\left(v_{\gamma}\right), G_{\gamma}^{-1}\left(v_{\gamma}\right) g_{\gamma}\left(G_{\gamma}^{-1}\left(v_{\gamma}\right)\right)\right\rangle \geq \frac{(p-2)(6-p)}{22-3 p} \int_{\mathbb{R}^{N}}\left|\nabla v_{\gamma}\right|^{2} d x
$$

Hence, we can deduce that

$$
\left\|\nabla v_{\gamma}\right\|_{2}^{2} \leq \frac{p(22-3 p)}{(p-2)(6-p)} m
$$

and

$$
\left\|v_{\gamma}\right\|_{2^{*}} \leq S^{-\frac{1}{2}}\left\|\nabla v_{\gamma}\right\|_{2} \leq \sqrt{\frac{p m(22-3 p)}{S(p-2)(6-p)}}
$$


Then, by the same proof as Proposition 3.6 of [20], we can deduce that there exists a constant $K>0$ independent of $\gamma$ such that $\left\|v_{\gamma}\right\|_{\infty} \leq K$. If $p \leq 4 \alpha$, let $\gamma_{0}:=\frac{p-2}{32 \alpha^{2}(4 \alpha-p)(2 K)^{2(2 \alpha-1)}}$, we have

$$
\left\|u_{\gamma}\right\|_{\infty}=\left\|G_{\gamma}^{-1}\left(v_{\gamma}\right)\right\| \leq 2\left\|v_{\gamma}\right\|_{\infty} \leq 2 K \leq\left(\frac{p-2}{32 \alpha^{2} \gamma(4 \alpha-p)}\right)^{\frac{1}{2(2 \alpha-1)}} \quad \text { for all } \gamma \in\left(0, \gamma_{0}\right)
$$

If $p \geq 4 \alpha$, let $\gamma_{0}:=\frac{p-2}{32 \alpha^{2}(6-p)(2 K)^{2(2 \alpha-1)}}$, we get

$$
\left\|u_{\gamma}\right\|_{\infty}=\left\|G_{\gamma}^{-1}\left(v_{\gamma}\right)\right\| \leq 2\left\|v_{\gamma}\right\|_{\infty} \leq 2 K \leq\left(\frac{p-2}{32 \alpha^{2} \gamma(6-p)}\right)^{\frac{1}{2(2 \alpha-1)}} \quad \text { for all } \gamma \in\left(0, \gamma_{0}\right)
$$

Hence, we can deduce that $g_{\gamma}\left(u_{\gamma}\right)=\sqrt{1+2 \alpha^{2} \gamma\left|u_{\gamma}\right|^{2(2 \alpha-1)}}$ if $\gamma \in\left(0, \gamma_{0}\right)$ and so $u_{\gamma}=G_{\gamma}^{-1}\left(v_{\gamma}\right)$ is a positive solution of (1).

\author{
Acknowledgements \\ The authors express their gratitude to the referees for valuable comments and suggestions. \\ Funding \\ Y.M. Zhang was supported by the Natural Science Foundation of China under grant numbers 11771127 and the \\ Fundamental Research Funds for the Central Universities (WUT: 2018IB014).
}

\title{
Availability of data and materials
}

Data sharing not applicable to this article as no data sets were generated or analyzed during the current study.

\section{Competing interests}

The authors declare that they have no competing interests.

\section{Authors' contributions}

All authors carried out the theoretical studies, participated in the design of the study and drafted the manuscript. All author read and approved the final manuscript.

\section{Publisher's Note}

Springer Nature remains neutral with regard to jurisdictional claims in published maps and institutional affiliations.

Received: 11 January 2019 Accepted: 13 March 2019 Published online: 18 March 2019

\section{References}

1. Adachi, S., Shibata, M., Watanabe, T.: Blow-up phenomena and asymptotic profiles of ground states of quasilinear elliptic equations with $\mathrm{H}^{1}$-supercritical nonlinearities. J. Differ. Equ. 256, 1492-1514 (2014)

2. Adachi, S., Watanabe, T.: G-invariant positive solutions for a quasilinear Schrödinger equation. Adv. Differ. Equ. 16, 3/4 (2011)

3. Adachi, S., Watanabe, T.: Asymptotic properties of ground states of quasilinear Schrödinger equations with $\mathrm{H}^{1}$-subcritical exponent. Adv. Nonlinear Stud. 12, 255-279 (2012)

4. Adachi, S., Watanabe, T.: Uniqueness of the ground state solutions of quasilinear Schrödinger equations. Nonlinear Anal. 75, 819-833 (2012)

5. Alves, C.O., Wang, Y.J., Shen, Y.T.: Soliton solutions for a class of quasilinear Schrödinger equations with a parameter. J. Differ. Equ. 259, 318-343 (2015)

6. Berestycki, H., Lions, P.L.: Nonlinear scalar field equations, I existence of a ground state. Arch. Ration. Mech. Anal. 82(4), 313-345 (1983)

7. Biemans, J., Platania, A., Saueressig, F.: Renormalization group fixed points of foliated gravity-matter systems. J. High Energy Phys. 05, 093 (2017)

8. Biemans, J., Platania, A., Saueressig, F.: Quantum gravity on foliated spacetimes - asymptotically safe and sound. Phys. Rev. D 95, 086013 (2017)

9. Colin, M., Jeanjean, L.: Solutions for a quasilinear Schrödinger equation: a dual approach. Nonlinear Anal. 56, 213-226 (2004)

10. Jeanjean, L., Tanaka, K.: A remark in least energy solutions in $\mathbb{R}^{N}$. Proc. Am. Math. Soc. 131, 2399-2408 (2003)

11. Kurihara, S.: Large-amplitude quasi-solitons in superfluid films. J. Phys. Soc. Jpn. 50, 3262-3267 (1981)

12. Li, Z.X., Zhang, Y.M.: Solutions for a class of quasilinear Schrödinger equations with critical Sobolev exponents. J. Math. Phys. 58, $021501(2017)$

13. Lions, P.L.: The concentration-compactness principle in the calculus of varations, the locally compact case, part I and part II. Rev. Mat. Iberoam. 1, 145-201, 223-283 (1985) 
14. Liu, J.Q., Wang, Y.Q., Wang, Z.Q.: Soliton solutions for quasilinear Schrödinger equations, II. J. Differ. Equ. 187, 473-493 (2003)

15. Liu, J.Q., Wang, Z.Q.: Soliton solutions for quasilinear Schrödinger equations, I. Proc. Am. Math. Soc. 131, 441-448 (2003)

16. Liu, X.Q., Liu, J.Q., Wang, Z.Q.: Ground states for quasilinear Schrödinger equations with critical growth. Calc. Var. Partial Differ. Equ. 46, 641-669 (2013)

17. Scapellato, A.: Homogeneous Herz spaces with variable exponents and regularity results. Electron. J. Qual. Theory Differ. Equ. 2018, 82 (2018)

18. Scapellato, A.: Regularity of solutions to elliptic equations on Herz spaces with variable exponents. Bound. Value Probl. 2019, 2 (2019)

19. Shen, Y.T., Wang, Y.J.: Soliton solutions for generalized quasilinear Schrödinger equations. Nonlinear Anal. 80, 194-201 (2013)

20. Wang, Y.J., Shen, Y.T.: Existence and asymptotic behavior of positive solutions for a class of quasilinear Schrödinger equations. Adv. Nonlinear Stud. 18(1), 131-150 (2017)

21. Zeng, X.Y., Zhang, Y.M.: Existence and asymptotic behavior for the ground state of quasilinear elliptic equation. Adv. Nonlinear Stud. 18(4), 725-744 (2018)

Submit your manuscript to a SpringerOpen ${ }^{\circ}$ journal and benefit from:

- Convenient online submission

- Rigorous peer review

- Open access: articles freely available online

- High visibility within the field

- Retaining the copyright to your article

Submit your next manuscript at $\gg$ springeropen.com 